\title{
DE RHAM COHOMOLOGY OF LOCAL COHOMOLOGY MODULES: THE GRADED CASE
}

\author{
TONY J. PUTHENPURAKAL
}

\begin{abstract}
Let $K$ be a field of characteristic zero, and let $R=K\left[X_{1}, \ldots, X_{n}\right]$. Let $A_{n}(K)=K\left\langle X_{1}, \ldots, X_{n}, \partial_{1}, \ldots, \partial_{n}\right\rangle$ be the $n$th Weyl algebra over $K$. We consider the case when $R$ and $A_{n}(K)$ are graded by giving $\operatorname{deg} X_{i}=\omega_{i}$ and $\operatorname{deg} \partial_{i}=-\omega_{i}$ for $i=1, \ldots, n$ (here $\omega_{i}$ are positive integers). Set $\omega=\sum_{k=1}^{n} \omega_{k}$. Let $I$ be a graded ideal in $R$. By a result due to Lyubeznik the local cohomology modules $H_{I}^{i}(R)$ are holonomic $\left(A_{n}(K)\right)$-modules for each $i \geq 0$. In this article we prove that the de Rham cohomology modules $H^{*}\left(\partial ; H_{I}^{*}(R)\right)$ are concentrated in degree $-\omega$; that is, $H^{*}\left(\partial ; H_{I}^{*}(R)\right)_{j}=0$ for $j \neq-\omega$. As an application when $A=R /(f)$ is an isolated singularity, we relate $H^{n-1}\left(\partial ; H_{(f)}^{1}(R)\right)$ to $H^{n-1}(\partial(f) ; A)$, the $(n-1)$ th Koszul cohomology of $A$ with respect to $\partial_{1}(f), \ldots, \partial_{n}(f)$.
\end{abstract}

Let $K$ be a field of characteristic zero, and let $R=K\left[X_{1}, \ldots, X_{n}\right]$. We consider $R$ graded with $\operatorname{deg} X_{i}=\omega_{i}$ for $i=1, \ldots, n$; here $\omega_{i}$ are positive integers. Set $\mathfrak{m}=\left(X_{1}, \ldots, X_{n}\right)$. Let $I$ be a graded ideal in $R$. The local cohomology modules $H_{I}^{*}(R)$ are clearly graded $R$-modules. Let $A_{n}(K)=K\left\langle X_{1}, \ldots, X_{n}\right.$, $\left.\partial_{1}, \ldots, \partial_{n}\right\rangle$ be the $n$th Weyl algebra over $K$. By a result due to Lyubeznik (see [3, Section 2.2.d]), the local cohomology modules $H_{I}^{i}(R)$ are holonomic $\left(A_{n}(K)\right)$-modules for each $i \geq 0$. We can consider $A_{n}(K)$ graded by giving $\operatorname{deg} \partial_{i}=-\omega_{i}$ for $i=1, \ldots, n$.

Let $N$ be a graded left $\left(A_{n}(K)\right)$-module. Now $\partial=\partial_{1}, \ldots, \partial_{n}$ are pairwise commuting $K$-linear maps, so we can consider the de Rham complex $K(\partial ; N)$. Notice that the de Rham cohomology modules $H^{*}(\partial ; N)$ are in general only graded $K$-vector spaces. They are finite-dimensional if $N$ is holonomic (see [1, Chapter 1, Theorem 6.1]). In particular, $H^{*}\left(\partial ; H_{I}^{*}(R)\right)$ are finite-dimensional graded $K$-vector spaces.

Our first result is as follows.

Received August 29, 2013. Accepted February 5, 2014.

First published online January 26, 2015.

2010 Mathematics Subject Classification. Primary 13D45; Secondary 13N10.

(C) 2015 by The Editorial Board of the Nagoya Mathematical Journal 
TheOREM 1. Let I be a graded ideal in $R$. Set $\omega=\sum_{i=1}^{n} \omega_{i}$. Then the de Rham cohomology modules $H^{*}\left(\partial_{1}, \ldots, \partial_{n} ; H_{I}^{*}(R)\right)$ are concentrated in degree $-\omega$; that is,

$$
H^{*}\left(\partial_{1}, \ldots, \partial_{n} ; H_{I}^{*}(R)\right)_{j}=0, \quad \text { for } j \neq-\omega .
$$

We give an application of Theorem 1. Let $f$ be a homogeneous polynomial in $R$, with $A=R /(f)$ an isolated singularity; that is, $A_{P}$ is regular for all homogeneous prime ideals $P \neq \mathfrak{m}$. Let $H^{i}(\partial(f) ; A)$ be the $i$ th Koszul cohomology of $A$ with respect to $\partial_{1}(f), \ldots, \partial_{n}(f)$. We show the following.

Theorem 2 (with hypotheses as above). There exists a filtration $\mathcal{F}=$ $\left\{\mathcal{F}_{\nu}\right\}_{\nu \geq 0}$ consisting of $K$-subspaces of $H^{n-1}\left(\partial ; H_{(f)}^{1}(R)\right)$ with $\mathcal{F}_{\nu}=H^{n-1}(\partial ;$ $\left.H_{(f)}^{1}(R)\right)$ for $\nu \gg 0, \mathcal{F}_{\nu} \supseteq F_{\nu-1}$, and $\mathcal{F}_{0}=0$ and injective $K$-linear maps

$$
\eta_{\nu}: \frac{\mathcal{F}_{\nu}}{\mathcal{F}_{\nu-1}} \rightarrow H^{n-1}(\partial(f) ; A)_{(\nu+1) \operatorname{deg} f-\omega}
$$

The techniques used in this theorem are generalized in [6] to show that $H^{i}\left(\partial ; H_{(f)}^{1}(R)\right)=0$ for $1<i<n-1$ and $H^{1}\left(\partial ; H_{(f)}^{1}(R)\right) \cong K$. There is no software to compute de Rham cohomology of an $\left(A_{n}(K)\right)$-module $M$. As an application of Theorem 2, we prove the following.

EXAMPLE 0.1. Let $R=K\left[X_{1}, \ldots, X_{n}\right]$, and let $f=X_{1}^{2}+X_{2}^{2}+\cdots+$ $X_{n-1}^{2}+X_{n}^{m}$ with $m \geq 2$. Then

(1) if $m$ is odd, then $H^{n-1}\left(\partial ; H_{(f)}^{1}(R)\right)=0$;

(2) if $m$ is even, then

(a) if $n$ is odd, then $H^{n-1}\left(\partial ; H_{(f)}^{1}(R)\right)=0$, and

(b) if $n$ is even, then $\operatorname{dim}_{K} H^{n-1}\left(\partial ; H_{(f)}^{1}(R)\right) \leq 1$.

We now describe in brief the contents of this article. In Section 1 we discuss a few preliminaries that we need. In Section 2 we introduce the concept of generalized Eulerian modules. In Section 3 we give a proof of Theorem 1. In Section 4 we give an outline of proof of Theorem 2. In Section 5 we prove Theorem 2. In Section 6 we give a proof of Example 0.1.

\section{$\S 1$. Preliminaries}

In this section we discuss a few preliminary results that we need.

REMARK 1.1. Although all the results are stated for de Rham cohomology of an $\left(A_{n}(K)\right)$-module $M$, we will actually work with de Rham homology. 
Note that $H_{i}(\partial, M)=H^{n-i}(\partial, M)$ for any $\left(A_{n}(K)\right)$-module $M$. Let $S=$ $K\left[\partial_{1}, \ldots, \partial_{n}\right]$. Consider it as a subring of $A_{n}(K)$. Then note that $H_{i}(\partial, M)$ is the $i$ th Koszul homology module of $M$ with respect to $\partial$.

1.2. Let $M$ be a holonomic $\left(A_{n}(K)\right)$-module. Then for the case where $i=$ 0,1 , the de Rham homology modules $H_{i}\left(\partial_{n}, M\right)$ are holonomic $\left(A_{n-1}(K)\right)$ modules (see [1, Theorem 6.2]).

The following result is well known (see [2, Corollary 1.6.13]).

Lemma 1.3. Let $\partial=\partial_{r}, \partial_{r+1}, \ldots, \partial_{n}$, and let $\partial^{\prime}=\partial_{r+1}, \ldots, \partial_{n}$. Let $M$ be a left $\left(A_{n}(K)\right)$-module. For each $i \geq 0$ there exists an exact sequence

$$
0 \rightarrow H_{0}\left(\partial_{r} ; H_{i}\left(\partial^{\prime} ; M\right)\right) \rightarrow H_{i}(\partial ; M) \rightarrow H_{1}\left(\partial_{r} ; H_{i-1}\left(\partial^{\prime} ; M\right)\right) \rightarrow 0
$$

\section{§2. Generalized Eulerian modules}

Consider the Eulerian operator

$$
\mathcal{E}_{n}=\omega_{1} X_{1} \partial_{1}+\omega_{2} X_{2} \partial_{2}+\cdots+\omega_{n} X_{n} \partial_{n}
$$

If $r \in R$ is homogeneous, then recall that $\mathcal{E}_{n} r=(\operatorname{deg} r) \cdot r$. Note that degree of $\mathcal{E}_{n}$ is zero.

Let $M$ be a graded $\left(A_{n}(K)\right)$-module. If $m$ is homogeneous, we set $|m|=$ $\operatorname{deg} m$. We say that $M$ is Eulerian $\left(A_{n}(K)\right)$-module if $\mathcal{E}_{n} m=|m| \cdot m$ for each homogeneous $m \in M$. This notion was discovered by Ma and Zhang (see their excellent paper [4]). They prove that local cohomology modules $H_{I}^{*}(R)$ are Eulerian $\left(A_{n}(K)\right)$-modules (see [4, Theorem 5.3]). In fact, they prove this when $R$ is standard graded. The same proof can be adapted to prove the general case.

It can easily be seen that if $M$ is an Eulerian $\left(A_{n}(K)\right)$-module, then so are each graded submodule and graded quotient of $M$. However, extensions of Eulerian modules need not be Eulerian (see [4, Remark 3.6]). To rectify this, we introduce the following notion. A graded $\left(A_{n}(K)\right)$-module $M$ is said to be generalized Eulerian if for a homogeneous element $m$ of $M$ there exists a positive integer $a$ (here $a$ may depend on $m$ ) such that

$$
\left(\mathcal{E}_{n}-|m|\right)^{a} m=0
$$

We now prove that the class of generalized Eulerian modules is closed under extensions. 
Proposition 2.1. Let $0 \rightarrow M_{1} \stackrel{\alpha_{1}}{\rightarrow} M_{2} \stackrel{\alpha_{2}}{\rightarrow} M_{3} \rightarrow 0$ be a short exact sequence of graded $\left(A_{n}(K)\right)$-modules. Then the following are equivalent:

(1) $M_{2}$ is generalized Eulerian,

(2) $M_{1}$ and $M_{3}$ are generalized Eulerian.

Proof. The assertion (1) $\Longrightarrow(2)$ is clear. We prove $(2) \Longrightarrow(1)$. Let $m \in M_{2}$ be homogeneous. Because $M_{3}$ is generalized Eulerian, we have

$$
\left(\mathcal{E}_{n}-|m|\right)^{b} \alpha_{2}(m)=0 \quad \text { for some } b \geq 1 .
$$

Set $v_{2}=\left(\mathcal{E}_{n}-|m|\right)^{b} m \in M_{2}$. Because $\alpha_{2}$ is $\left(A_{n}(K)\right)$-linear, we get $\alpha_{2}\left(v_{2}\right)=0$. So $v_{2}=\alpha_{1}\left(v_{1}\right)$ for some $v_{1} \in M_{1}$. Note that $\operatorname{deg} v_{1}=$ $\operatorname{deg} v_{2}=|m|$. Because $M_{1}$ is generalized Eulerian, we have

$$
\left(\mathcal{E}_{n}-|m|\right)^{a} v_{1}=0 \quad \text { for some } a \geq 1 .
$$

Because $\alpha_{1}$ is $\left(A_{n}(K)\right)$-linear, we get $\left(\mathcal{E}_{n}-|m|\right)^{a} v_{2}=0$. It follows that

$$
\left(\mathcal{E}_{n}-|m|\right)^{a+b} m=0 .
$$

If $M$ is a graded $\left(A_{n}(K)\right)$-module, then for $l \in \mathbb{Z}$ the module $M(l)$ denotes the shift of $M$ by $l$; that is, $M(l)_{n}=M_{n+l}$ for all $n \in \mathbb{Z}$. The following result was proved for Eulerian $\left(A_{n}(K)\right)$-modules in [4, Remark 2.5].

Proposition 2.2. Let $M$ be a nonzero generalized Eulerian $\left(A_{n}(K)\right)$ module. Then for $l \neq 0$, the module $M(l)$ is not a generalized Eulerian $\left(A_{n}(K)\right)$-module.

Proof. Suppose that $M(l)$ is a generalized Eulerian $\left(A_{n}(K)\right)$-module for some $l \neq 0$. Let $m \in M$ be homogeneous of degree $r$ and nonzero. Because $M$ is generalized Eulerian $\left(A_{n}(K)\right)$-module, we have

$$
\left(\mathcal{E}_{n}-r\right)^{a} m=0 \quad \text { for some } a \geq 1 \text {. }
$$

We may assume that $\left(\mathcal{E}_{n}-r\right)^{a-1} m \neq 0$. Now $m \in M(l)_{r-l}$. Because $M(l)$ is generalized Eulerian, we get

$$
\left(\mathcal{E}_{n}-r+l\right)^{b} m=0 \quad \text { for some } b \geq 1 .
$$

Notice that

$$
0=\left(\mathcal{E}_{n}-r+l\right)^{b} m=\left(l^{b}+\sum_{i=1}^{b}\left(\begin{array}{l}
b \\
i
\end{array}\right) l^{b-i}\left(\mathcal{E}_{n}-r\right)^{i}\right) m .
$$


Multiply the term on the left by $\left(\mathcal{E}_{n}-r\right)^{a-1}$. We obtain

$$
l^{b}\left(\mathcal{E}_{n}-r\right)^{a-1} m=0 .
$$

Because $l \neq 0$, we get $\left(\mathcal{E}_{n}-r\right)^{a-1} m=0$, a contradiction.

\section{$\S 3$. Proof of Theorem 1}

In this section we prove Theorem 1. Notice that $H_{I}^{i}(R)$ are Eulerian $\left(A_{n}(K)\right)$-modules for all $i \geq 0$. Hence, Theorem 1 follows from the following more general result.

TheOREM 3.1. Let $M$ be a generalized Eulerian $\left(A_{n}(K)\right)$-module. Then $H_{i}(\partial ; M)$ is concentrated in degree $-\omega=-\sum_{k=1}^{n} \omega_{k}$.

Before proving Theorem 3.1, we need to prove a few preliminary results.

Proposition 3.2. Let $M$ be a generalized Eulerian $\left(A_{n}(K)\right)$-module. Then for $i=0,1$, the $\left(A_{n-1}(K)\right)$-modules $H_{i}\left(\partial_{n} ; M\right)\left(-\omega_{n}\right)$ are generalized Eulerian.

Proof. Clearly, $H_{i}\left(\partial_{n} ; M\right)\left(-\omega_{n}\right)$ are $\left(A_{n-1}(K)\right)$-modules for $i=0,1$. We have an exact sequence of $\left(A_{n-1}(K)\right)$-modules

$$
0 \rightarrow H_{1}\left(\partial_{n} ; M\right) \rightarrow M\left(\omega_{n}\right) \stackrel{\partial_{n}}{\longrightarrow} M \rightarrow H_{0}\left(\partial_{n} ; M\right) \rightarrow 0
$$

Note that $H_{1}\left(\partial_{n} ; M\right)\left(-\omega_{n}\right) \subset M$. Let $\xi \in H_{1}\left(\partial_{n} ; M\right)\left(-\omega_{n}\right)$ be homogeneous. As $M$ is generalized Eulerian, we have

$$
\left(\mathcal{E}_{n}-|\xi|\right)^{a} \xi=0 \quad \text { for some } a \geq 1
$$

Notice that $\mathcal{E}_{n}=\mathcal{E}_{n-1}+\omega_{n} X_{n} \partial_{n}$. Also note that $X_{n} \partial_{n}$ commutes with $\mathcal{E}_{n-1}$. Thus,

$$
0=\left(\mathcal{E}_{n-1}-|\xi|+\omega_{n} X_{n} \partial_{n}\right)^{a} \xi=\left(\left(\mathcal{E}_{n-1}-|\xi|\right)^{a}+(*) X_{n} \partial_{n}\right) \xi
$$

Because $\partial_{n} \xi=0$, we get $\left(\mathcal{E}_{n-1}-|\xi|\right)^{a} \xi=0$. It follows that $H_{1}\left(\partial_{n} ; M\right)\left(-\omega_{n}\right)$ is a generalized Eulerian $\left(A_{n-1}(K)\right)$-module.

Let $\xi \in H_{0}\left(\partial_{n} ; M\right)\left(-\omega_{n}\right)$ be homogeneous of degree $r$. Then $\xi=\alpha+\partial_{n} M$, where $\alpha \in M_{r-\omega_{n}}$. Because $M$ is generalized Eulerian, we get

$$
\left(\mathcal{E}_{n}-r+\omega_{n}\right)^{a} \alpha=0 \quad \text { for some } a \geq 1
$$


Notice that $\mathcal{E}_{n}=\mathcal{E}_{n-1}+\omega_{n} X_{n} \partial_{n}=\mathcal{E}_{n-1}+\omega_{n} \partial_{n} X_{n}-\omega_{n}$, so $\mathcal{E}_{n}-r+\omega_{n}=$ $\mathcal{E}_{n-1}-r+\omega_{n} \partial_{n} X_{n}$. Notice that $\partial_{n} X_{n}$ commutes with $\mathcal{E}_{n-1}$. Thus,

$$
0=\left(\mathcal{E}_{n-1}-r+\omega_{n} \partial_{n} X_{n}\right)^{a} \alpha=\left(\mathcal{E}_{n-1}-r\right)^{a} \alpha+\partial_{n} \cdot * \alpha .
$$

Going $\bmod \partial_{n} M$, we get

$$
\left(\mathcal{E}_{n-1}-r\right)^{a} \xi=0 .
$$

It follows that $H_{0}\left(\partial_{n} ; M\right)\left(-\omega_{n}\right)$ is a generalized Eulerian $\left(A_{n-1}(K)\right)$-module.

REMARK 3.3. If $M$ is Eulerian, then the same proof shows that $H_{i}\left(\partial_{n} ; M\right)\left(-\omega_{n}\right)$ is an Eulerian $\left(A_{n-1}(K)\right)$-module for $i=0,1$. However, as the proof of the following theorem shows, we can prove only that $H_{1}\left(\partial_{n-1}, \partial_{n} ; M\right)\left(-\omega_{n-1}-\omega_{n}\right)$ is a generalized Eulerian $\left(A_{n-1}(K)\right)$-module.

Proposition 3.4. Let $M$ be a generalized Eulerian $\left(A_{n}(K)\right)$-module. Let $\partial=\partial_{i}, \partial_{i+1}, \ldots, \partial_{n} ;$ here $i \geq 2$. Then for each $j \geq 0$ the de Rham homology module

$$
H_{j}(\partial ; M)\left(-\sum_{k=i}^{n} \omega_{k}\right)
$$

is a generalized Eulerian $\left(A_{i-1}(K)\right)$-module.

Proof. We prove this result by descending induction on $i$. For $i=n$, the result holds by Proposition 3.2. Set $\partial^{\prime}=\partial_{i+1}, \ldots, \partial_{n}$. By induction hypothesis $H_{j}\left(\partial^{\prime} ; M\right)\left(-\sum_{k=i+1}^{n} \omega_{k}\right)$ is generalized Eulerian $\left(A_{i}(K)\right)$-module. By Proposition 3.2 again, for $l=0,1$ and for each $j \geq 0$,

$$
H_{l}\left(\partial_{i} ; H_{j}\left(\partial^{\prime} ; M\right)\left(-\sum_{k=i+1}^{n} \omega_{k}\right)\right)\left(-\omega_{i}\right)=H_{l}\left(\partial_{i} ; H_{j}\left(\partial^{\prime} ; M\right)\right)\left(-\sum_{k=i}^{n} \omega_{k}\right)
$$

is generalized Eulerian. By Lemma 1.3 we have the exact sequence

$$
0 \rightarrow H_{0}\left(\partial_{i} ; H_{j}\left(\partial^{\prime} ; M\right)\right) \rightarrow H_{j}(\partial ; M) \rightarrow H_{1}\left(\partial_{i} ; H_{j-1}\left(\partial^{\prime} ; M\right)\right) \rightarrow 0 .
$$

The modules at the left and right end become generalized Eulerian after shifting by $-\sum_{k=i}^{n} \omega_{k}$. By Proposition 2.1 it follows that for each $j \geq 0$ the de Rham homology module

$$
H_{j}(\partial ; M)\left(-\sum_{k=i}^{n} \omega_{k}\right)
$$

is a generalized Eulerian $\left(A_{i-1}(K)\right)$-module. 
We now consider the case when $n=1$.

Proposition 3.5. Let $M$ be a generalized Eulerian $\left(A_{1}(K)\right)$-module. Then for $l=0,1$ the modules $H_{l}\left(\partial_{1} ; M\right)$ are concentrated in degree $-\omega_{1}$.

Proof. We have an exact sequence of $K$-vector spaces

$$
0 \rightarrow H_{1}\left(\partial_{1} ; M\right) \rightarrow M\left(\omega_{1}\right) \stackrel{\partial_{1}}{\rightarrow} M \rightarrow H_{0}\left(\partial_{1} ; M\right) \rightarrow 0 .
$$

Let $\xi \in H_{1}\left(\partial_{1} ; M\right)\left(-\omega_{1}\right)$ be homogeneous and nonzero. Because $\xi \in M$, we have

$$
\left(\omega_{1} X_{1} \partial_{1}-|\xi|\right)^{a} \xi=0 \text { for some } a \geq 1 .
$$

Notice that $\left(\omega_{1} X_{1} \partial_{1}-|\xi|\right)^{a}=(*) \partial_{1}+(-1)^{a}|\xi|^{a}$. Because $\partial_{1} \xi=0$, we get $(-1)^{a}|\xi|^{a} \xi=0$. Because $\xi \neq 0$, we get $|\xi|=0$. It follows that $H_{1}\left(\partial_{1} ; M\right)$ is concentrated in degree $-\omega_{1}$.

Let $\xi \in H_{0}\left(\partial_{1}, M\right)$ be nonzero and homogeneous of degree $r$. Let $\xi=$ $\alpha+\partial_{1} M$, where $\alpha \in M_{r}$. Because $M$ is generalized Eulerian, we get

$$
\left(\omega_{1} X_{1} \partial_{1}-r\right)^{a} \alpha=0 \text { for some } a \geq 1
$$

Notice that $\omega_{1} X_{1} \partial_{1}=\omega_{1} \partial_{1} X_{1}-\omega_{1}$, so we have

$$
0=\left(\omega_{1} \partial_{1} X_{1}-\left(r+\omega_{1}\right)\right)^{a} \alpha=\left(\partial_{1} *+(-1)^{a}\left(r+\omega_{1}\right)^{a}\right) \alpha .
$$

In $M / \partial_{1} M$, we have $(-1)^{a}\left(r+\omega_{1}\right)^{a} \xi=0$. Because $\xi \neq 0$, we get $r=-\omega_{1}$. It follows that $H_{0}\left(\partial_{1} ; M\right)$ is concentrated in degree $-\omega_{1}$.

We now give the following.

Proof of Theorem 3.1. Set $\partial^{\prime}=\partial_{2}, \ldots, \partial_{n}$. By Proposition 3.4, $N_{j}=$ $H_{j}\left(\partial^{\prime} ; M\right)\left(-\sum_{k=2}^{n} \omega_{k}\right)$ is a generalized Eulerian $\left(A_{1}(K)\right)$-module, for each $j \geq 0$. We use exact sequence in Lemma 1.3 and shift it by $-\sum_{k=2}^{n} \omega_{k}$ to obtain an exact sequence

$$
0 \rightarrow H_{0}\left(\partial_{1}, N_{j}\right) \rightarrow H_{j}(\partial ; M)\left(-\sum_{k=2}^{n} \omega_{k}\right) \rightarrow H_{1}\left(\partial_{1}, N_{j-1}\right) \rightarrow 0
$$

for each $j \geq 0$. By Proposition 3.5, the modules on the left and right of the above exact sequence are concentrated in degree $-\omega_{1}$. It follows that for each $j \geq 0$ the $K$-vector space $H_{j}(\partial ; M)$ is concentrated in degree $-\omega=$ $-\sum_{k=1}^{n} \omega_{k}$. 


\section{$\S 4$. Outline of proof of Theorem 2}

The proof of Theorem 2 is a bit long and has a lot of technical details. For the convenience of the reader, we give an outline of the proof.

4.1. By [5, Lemma 2.7], we have $H_{1}\left(\partial, R_{f}\right) \cong H_{1}\left(\partial, H_{(f)}^{1}(R)\right)$. Thus, it is sufficient to work with $H_{1}\left(\partial, R_{f}\right)$ in order to prove Theorem 2 . We consider elements of $R_{f}^{m}$ as column vectors. For $x \in R_{f}^{m}$, we write $x=\left(x_{1}, \ldots, x_{m}\right)^{\prime}$; here $/$ indicates "transpose".

4.2. Let $\xi \in R_{f}^{m} \backslash R^{m}$. The element $\left(a_{1} / f^{i}, a_{2} / f^{i}, \ldots, a_{m} / f^{i}\right)^{\prime}$, with $a_{j} \in R$ for all $j$, is said to be a normal form of $\xi$ if

(1) $\xi=\left(a_{1} / f^{i}, a_{2} / f^{i}, \ldots, a_{m} / f^{i}\right)^{\prime}$,

(2) $f$ does not divide $a_{j}$ for some $j$, and

(3) $i \geq 1$.

It can easily be shown that the normal form of $\xi$ exists and is unique (see Proposition 5.1). Let $\left(a_{1} / f^{i}, a_{2} / f^{i}, \ldots, a_{m} / f^{i}\right)^{\prime}$ be the normal form of $\xi$. Set $L(\xi)=i$. Notice that $L(\xi) \geq 1$.

4.3. Construction of a function $\theta: Z_{1}\left(\partial, R_{f}\right) \backslash R^{n} \rightarrow H_{1}(\partial(f) ; A)$

Let $\xi \in Z_{1}\left(\partial, R_{f}\right) \backslash R^{n}$. Let $\left(a_{1} / f^{i}, a_{2} / f^{i}, \ldots, a_{n} / f^{i}\right)^{\prime}$ be the normal form of $\xi$. Thus, we have $\sum_{j=1}^{n} \partial / \partial X_{j}\left(a_{j} / f^{i}\right)=0$, so we have

$$
\frac{1}{f^{i}}\left(\sum_{j=1}^{n} \frac{\partial a_{j}}{\partial X_{j}}\right)-\frac{i}{f^{i+1}}\left(\sum_{j=1}^{n} a_{j} \frac{\partial f}{\partial X_{j}}\right)=0 .
$$

It follows that

$$
f \text { divides } \sum_{j=1}^{n} a_{j} \frac{\partial f}{\partial X_{j}} .
$$

Thus, $\left(\overline{a_{1}}, \ldots, \overline{a_{n}}\right)^{\prime} \in Z_{1}(\partial(f) ; A)$. We set

$$
\theta(\xi)=\left[\left(\overline{a_{1}}, \ldots, \overline{a_{n}}\right)^{\prime}\right] \in H_{1}(\partial(f) ; A) .
$$

REMARK 4.4. It can be shown that if $\xi \in Z_{1}\left(\partial, R_{f}\right)_{-\omega}$ is nonzero, then $\xi \notin R^{n}$ (see Section 5.2). If $L(\xi)=i$, then by Section 5.3 we have

$$
\theta(\xi) \in H_{1}(\partial(f) ; A)_{(i+1) \operatorname{deg} f-\omega} .
$$


The next result uses the fact that $A$ is an isolated singularity.

Proposition 4.5. If $\xi \in B_{1}\left(\partial, R_{f}\right)_{-\omega}$ is nonzero, then $\theta(\xi)=0$.

4.6. Let $\xi \in R_{f}^{m}$. We define $L(f)$ as follows.

Case 1: $\xi \in R_{f}^{m} \backslash R^{m}$. Let $\left(a_{1} / f^{i}, a_{2} / f^{i}, \ldots, a_{m} / f^{i}\right)^{\prime}$ be the normal form of $\xi$. Set $L(\xi)=i$. Notice that $L(\xi) \geq 1$ in this case.

Case 2: $\xi \in R^{m} \backslash\{0\}$. Set $L(\xi)=0$.

Case 3: $\xi=0$. Set $L(\xi)=-\infty$.

The following properties of the function $L$ can be easily verified.

Proposition 4.7 (with hypotheses as above). Let $\xi, \xi_{1}, \xi_{2} \in R_{f}^{m}$, and let $\alpha, \alpha_{1}, \alpha_{2} \in K$. Then we have the following:

(1) if $L\left(\xi_{1}\right)<L\left(\xi_{2}\right)$, then $L\left(\xi_{1}+\xi_{2}\right)=L\left(\xi_{2}\right)$;

(2) if $L\left(\xi_{1}\right)=L\left(\xi_{2}\right)$, then $L\left(\xi_{1}+\xi_{2}\right) \leq L\left(\xi_{2}\right)$;

(3) $L\left(\xi_{1}+\xi_{2}\right) \leq \max \left\{L\left(\xi_{1}\right), L\left(\xi_{2}\right)\right\}$;

(4) if $\alpha \in K^{*}$, then $L(\alpha \xi)=L(\xi)$;

(5) $L(\alpha \xi) \leq L(\xi)$ for all $\alpha \in K$;

(6) $L\left(\alpha_{1} \xi_{1}+\alpha_{2} \xi_{2}\right) \leq \max \left\{L\left(\xi_{1}\right), L\left(\xi_{2}\right)\right\}$;

(7) let $\xi_{1}, \ldots, \xi_{r} \in R_{f}^{m}$, and let $\alpha_{1}, \ldots, \alpha_{r} \in K$. Then

$$
L\left(\sum_{j=1}^{r} \alpha_{j} \xi_{j}\right) \leq \max \left\{L\left(\xi_{1}\right), L\left(\xi_{2}\right), \ldots, L\left(\xi_{r}\right)\right\}
$$

4.8. We now use the fact that $H_{1}\left(\partial, R_{f}\right)$ is concentrated in degree $-\omega=$ $-\sum_{k=1}^{n} \omega_{k}$. Thus,

$$
H_{1}\left(\partial, R_{f}\right)=H_{1}\left(\partial, R_{f}\right)_{-\omega}=\frac{Z_{1}\left(\partial, R_{f}\right)_{-\omega}}{B_{1}\left(\partial, R_{f}\right)_{-\omega}}
$$

Let $x \in H_{1}\left(\partial, R_{f}\right)$ be nonzero. Define

$$
L(x)=\min \left\{L(\xi) \mid x=[\xi], \text { where } \xi \in Z_{1}\left(\partial, R_{f}\right)_{-\omega}\right\}
$$

It can be shown that $L(x) \geq 1$. If $x=0$, then set

$$
L(0)=-\infty
$$


We now define a function

$$
\begin{aligned}
\widetilde{\theta}: H_{1}\left(\partial, R_{f}\right) & \rightarrow H_{1}(\partial(f) ; A) \\
x & \mapsto \begin{cases}\theta(\xi) & \text { if } x \neq 0, x=[\xi], \text { and } L(x)=L(\xi), \\
0 & \text { if } x=0 .\end{cases}
\end{aligned}
$$

It can be shown that $\widetilde{\theta}(x)$ is independent of choice of $\xi$ (see Proposition 5.6). Also note that if $L(x)=i$, then $\widetilde{\theta}(x) \in H_{1}(\partial(f) ; A)_{(i+1) \operatorname{deg} f-\omega}$.

4.9. We now construct a filtration $\mathcal{F}=\left\{\mathcal{F}_{\nu}\right\}_{\nu \geq 0}$ of $H_{1}\left(\partial, R_{f}\right)$. Set

$$
\mathcal{F}_{\nu}=\left\{x \in H_{1}\left(\partial, R_{f}\right) \mid L(x) \leq \nu\right\}
$$

In Section 5, we prove the following.

Proposition 4.10. We have the following:

(1) $\mathcal{F}_{\nu}$ is a $K$ subspace of $H_{1}\left(\partial, R_{f}\right)$,

(2) $\mathcal{F}_{\nu} \supseteq \mathcal{F}_{\nu-1}$ for all $\nu \geq 1$,

(3) $\mathcal{F}_{\nu}=H_{1}\left(\partial, R_{f}\right)$ for all $\nu \gg 0$,

(4) $\mathcal{F}_{0}=0$.

Let $\mathcal{G}=\bigoplus_{\nu \geq 1} \mathcal{F}_{\nu} / \mathcal{F}_{\nu-1}$. For $\nu \geq 1$, we define

$$
\begin{aligned}
\eta_{\nu}: \frac{\mathcal{F}_{\nu}}{\mathcal{F}_{\nu-1}} & \rightarrow H_{1}(\partial(f) ; A)_{(\nu+1) \operatorname{deg} f-\omega} \\
\xi & \mapsto \begin{cases}0 & \text { if } \xi=0, \\
\tilde{\theta}(x) & \text { if } \xi=x+\mathcal{F}_{\nu-1} \text { is nonzero. }\end{cases}
\end{aligned}
$$

It can be shown that $\eta_{\nu}(\xi)$ is independent of choice of $x$ (see Proposition 5.10). Finally we prove the following result.

THEOREM 4.11 (with notation as above). For all $\nu \geq 1$,

(1) $\eta_{\nu}$ is K-linear, and

(2) $\eta_{\nu}$ is injective.

\section{§5. Proof of Theorem 2}

In this section we give a proof of Theorem 2 with all details. The reader is advised to read the preceding section before reading this section.

We first prove the following. 
Proposition 5.1. Let $\xi \in R_{f}^{m} \backslash R^{m}$. Then a normal form of $\xi$ exists and is unique.

Proof. Existence: Let $\xi \in R_{f}^{m} \backslash R^{m}$. Let $\xi=\left(b_{1} / f^{i_{1}}, b_{2} / f^{i_{2}}, \ldots, b_{m} / f^{i_{m}}\right)^{\prime}$ with $f \nmid b_{j}$ if $b_{j} \neq 0$. Note that $i_{j} \leq 0$ is possible. Let

$$
i_{r}=\max \left\{i_{j} \mid i_{j} \geq 1 \text { and } b_{j} \neq 0\right\} .
$$

Notice that $i_{r} \geq 1$. Then

$$
\xi=\left(\frac{b_{1} f^{i_{r}-i_{1}}}{f^{i_{r}}}, \frac{b_{2} f^{i_{r}-i_{2}}}{f^{i_{r}}}, \ldots, \frac{b_{m} f^{i_{r}-i_{m}}}{f^{i_{r}}}\right)^{\prime} .
$$

Note that $f \nmid b_{r}$. Thus, the expression above is a normal form of $\xi$.

Uniqueness: Let $\left(a_{1} / f^{i}, \ldots, a_{m} / f^{i}\right)^{\prime}$ and $\left(b_{1} / f^{r}, \ldots, b_{m} / f^{r}\right)^{\prime}$ be two normal forms of $\xi$. We first assert that $i<r$ is not possible, for if this holds, then because $a_{j} / f^{i}=b_{j} / f^{r}$, we get $b_{j}=a_{j} f^{r-i}$, so $f \mid b_{j}$ for all $j$, a contradiction.

A similar argument shows that $i>r$ is not possible, so $i=r$. Thus, $a_{j}=b_{j}$ for all $j$. Thus, the normal form of $\xi$ is unique.

5.2. Let $\xi \in Z_{1}\left(\partial, R_{f}\right)_{-\omega}$ be nonzero. Let $\xi=\left(\xi_{1}, \ldots, \xi_{n}\right)^{\prime}$. Note that

$$
\xi \in\left(R_{f}\left(\omega_{1}\right) \oplus R_{f}\left(\omega_{2}\right) \oplus \cdots \oplus R_{f}\left(\omega_{n}\right)\right)_{-\omega} .
$$

It follows that

$$
\xi_{j} \in\left(R_{f}\right)_{-\sum_{k \neq j} \omega_{k}}
$$

It follows that $\xi \in R_{f}^{n} \backslash R^{n}$.

5.3. Let $\left(a_{1} / f^{i}, \ldots, a_{n} / f^{i}\right)^{\prime}$ be the normal form of $\xi$. Then

$$
\operatorname{deg} a_{j}=i \operatorname{deg} f-\sum_{k \neq j} \omega_{k} .
$$

In particular, going $\bmod f$, we get

$$
\overline{a_{j}} \in A\left(-\operatorname{deg} f+\omega_{j}\right)_{(i+1) \operatorname{deg} f-\omega} .
$$

Notice that $\operatorname{deg} \partial f / \partial X_{j}=\operatorname{deg} f-\omega_{j}$. It follows that

$$
\left(\overline{a_{1}}, \ldots, \overline{a_{n}}\right)^{\prime} \in Z_{1}(\partial(f) ; A)_{(i+1) \operatorname{deg} f-\omega} .
$$

Thus, $\theta(\xi) \in H_{1}(\partial(f) ; A)_{(i+1) \operatorname{deg} f-\omega}$. 
5.4. Let $\mathbb{K}=\mathbb{K}\left(\partial ; R_{f}\right)$ be the de Rham complex on $R_{f}$ written homologically, so

$$
\mathbb{K}=\cdots \rightarrow \mathbb{K}_{3} \stackrel{\phi_{3}}{\longrightarrow} \mathbb{K}_{2} \stackrel{\phi_{2}}{\longrightarrow} \mathbb{K}_{1} \stackrel{\phi_{1}}{\longrightarrow} \mathbb{K}_{0} \rightarrow 0
$$

Here $\mathbb{K}_{0}=R_{f}, \mathbb{K}_{1}=\bigoplus_{k=1}^{n} R_{f}\left(\omega_{k}\right)$,

$$
\mathbb{K}_{2}=\bigoplus_{1 \leq i<j \leq n} R_{f}\left(\omega_{i}+\omega_{j}\right), \quad \text { and } \quad \mathbb{K}_{3}=\bigoplus_{1 \leq i<j<l \leq n} R_{f}\left(\omega_{i}+\omega_{j}+\omega_{l}\right)
$$

Let $\mathbb{K}^{\prime}=\mathbb{K}(\partial(f) ; A)$ be the Koszul complex on $A$ with respect to $\partial f / \partial X_{1}$, $\ldots, \partial f / \partial X_{n}$. Thus,

$$
\mathbb{K}^{\prime}=\cdots \rightarrow \mathbb{K}_{3}^{\prime} \stackrel{\psi_{3}}{\longrightarrow} \mathbb{K}_{2}^{\prime} \stackrel{\psi_{2}}{\longrightarrow} \mathbb{K}_{1}^{\prime} \stackrel{\psi_{1}}{\longrightarrow} \mathbb{K}_{0}^{\prime} \rightarrow 0 .
$$

Here $\mathbb{K}_{0}^{\prime}=A, \mathbb{K}_{1}^{\prime}=\bigoplus_{k=1}^{n} A\left(-\operatorname{deg} f+\omega_{k}\right)$,

$$
\begin{aligned}
& \mathbb{K}_{2}^{\prime}=\bigoplus_{1 \leq i<j \leq n} A\left(-2 \operatorname{deg} f+\omega_{i}+\omega_{j}\right), \quad \text { and } \\
& \mathbb{K}_{3}^{\prime}=\bigoplus_{1 \leq i<j<l \leq n} A\left(-3 \operatorname{deg} f+\omega_{i}+\omega_{j}+\omega_{l}\right) .
\end{aligned}
$$

We now prove Proposition 4.5.

Proof of Proposition 4.5. Let $u \in B_{1}\left(\partial ; R_{f}\right)_{-\omega}$ be nonzero. Let $\xi \in$ $\left(\mathbb{K}_{2}\right)_{-\omega}$ be homogeneous, with $\phi_{2}(\xi)=u$. Let $\xi=\left(\xi_{i j} \mid 1 \leq i<j \leq n\right)^{\prime}$. Notice that

$$
\xi_{i j} \in R_{f}\left(\omega_{i}+\omega_{j}\right)_{-\omega}=\left(R_{f}\right)_{-\sum_{k \neq i, j} \omega_{k}}
$$

It follows that $\xi \in R_{f}^{\left(\begin{array}{c}n \\ 2\end{array}\right)} \backslash R^{\left(\begin{array}{c}n \\ 2\end{array}\right)}$. Set

$$
c=\min \left\{j \mid j=L(\xi) \text { where } \phi_{2}(\xi)=u \text { and } \xi \in\left(\mathbb{K}_{2}\right)_{-\omega} \text { is homogeneous }\right\} .
$$

Notice that $c \geq 1$. Let $\xi \in\left(\mathbb{K}_{2}\right)_{-\omega}$ be such that $L(\xi)=c$ and $\phi_{2}(\xi)=u$. Let $\left(b_{i j} / f^{c} \mid 1 \leq i<j \leq n\right)^{\prime}$ be the normal form of $\xi$. Let $u=\left(u_{1}, \ldots, u_{n}\right)^{\prime}$. Then for $l=1, \ldots, n$,

$$
u_{l}=\sum_{i<l} \frac{\partial}{\partial X_{i}}\left(\frac{b_{i l}}{f^{c}}\right)-\sum_{j>l} \frac{\partial}{\partial X_{j}}\left(\frac{b_{l j}}{f^{c}}\right) .
$$

So

$$
u_{l}=\frac{f}{f^{c+1}}\left(\sum_{i<l} \frac{\partial\left(b_{i l}\right)}{\partial X_{i}}-\sum_{j>l} \frac{\partial\left(b_{l j}\right)}{\partial X_{j}}\right)+\frac{c}{f^{c+1}}\left(-\sum_{i<l} b_{i l} \frac{\partial f}{\partial X_{i}}+\sum_{j>l} b_{l j} \frac{\partial f}{\partial X_{j}}\right) .
$$


Set

$$
v_{l}=c\left(-\sum_{i<l} b_{i l} \frac{\partial f}{\partial X_{i}}+\sum_{j>l} b_{l j} \frac{\partial f}{\partial X_{j}}\right)
$$

Therefore,

$$
u_{l}=\frac{f *+v_{l}}{f^{c+1}}
$$

Claim. $f \nmid v_{l}$ for some $l$. First assume the claim. Then $\left(\left(f *+v_{1}\right) / f^{c+1}\right.$, $\left.\ldots,\left(f *+v_{n}\right) / f^{c+1}\right)^{\prime}$ is the normal form of $u$. Thus,

$$
\theta(u)=\left[\left(\overline{v_{1}}, \ldots, \overline{v_{n}}\right)^{\prime}\right]=\left[\psi_{2}(-c \bar{b})\right]=0 .
$$

We now prove our claim. Suppose, if possible, that $f \mid v_{l}$ for all $l$. Then

$$
\psi_{2}(-c \bar{b})=\left(\overline{v_{1}}, \ldots, \overline{v_{l}}\right)^{\prime}=0
$$

so $-c b \in Z_{2}(\partial(f) ; A)$. Because $H_{2}(\partial(f) ; A)=0$, we get $-c b \in B_{2}(\partial(f) ; A)$. Thus, $-c \bar{b}=\psi_{3}(\bar{\gamma})$. Here

$$
\gamma=\left(\gamma_{i j l} \mid 1 \leq i<j<l \leq n\right)^{\prime}
$$

Thus,

$$
-c b_{i j}=\sum_{k<i<j} \gamma_{k i j} \frac{\partial f}{\partial X_{k}}-\sum_{i<k<j} \gamma_{i k j} \frac{\partial f}{\partial X_{k}}+\sum_{i<j<k} \gamma_{i j k} \frac{\partial f}{\partial X_{k}}+\alpha_{i j} f .
$$

We need to compute the degree of $\gamma_{i j l}$. Note that $\xi \in\left(\mathbb{K}_{2}\right)_{-\omega}$, so

$$
\frac{b_{i j}}{f^{c}} \in\left(R_{f}\left(\omega_{i}+\omega_{j}\right)\right)_{-\omega} .
$$

It follows that

$$
\operatorname{deg} b_{i j}=c \operatorname{deg} f-\omega+\omega_{i}+\omega_{j}
$$

It can be easily checked that

$$
\bar{b} \in\left(\mathbb{K}_{2}^{\prime}\right)_{(c+2) \operatorname{deg} f-\omega}
$$

SO

$$
\gamma \in\left(\mathbb{K}_{3}^{\prime}\right)_{(c+2) \operatorname{deg} f-\omega}
$$


It follows that

$$
\operatorname{deg} \gamma_{i j l}=(c-1) \operatorname{deg} f-\omega+\omega_{i}+\omega_{j}+\omega_{l}
$$

We first consider the case when $c=1$. Then by (5.4.1), we have $\alpha_{i j}=0$. Also,

$$
\operatorname{deg} \gamma_{i j l}=-\omega+\omega_{i}+\omega_{j}+\omega_{l}<0 \text { if } n>3,
$$

so if $n>3$, we get $\gamma_{i j l}=0$. Thus, $b=0$, so $\xi=0$, a contradiction.

We now consider the case when $n=3$. Note that $\gamma=\gamma_{123}$ is a constant. Thus,

$$
b=\left(\gamma \frac{\partial f}{\partial X_{3}},-\gamma \frac{\partial f}{\partial X_{2}}, \gamma \frac{\partial f}{\partial X_{3}}\right)^{\prime}
$$

A direct computation yields $u=0$, a contradiction.

We now consider the case when $c \geq 2$. Notice that by (5.4.1), we have

$$
\frac{-c b_{i j}}{f^{c}}=\frac{1}{f^{c}} \sum_{k<i<j} \gamma_{k i j} \frac{\partial f}{\partial X_{k}}-\frac{1}{f^{c}} \sum_{i<k<j} \gamma_{i k j} \frac{\partial f}{\partial X_{k}}+\frac{1}{f^{c}} \sum_{i<j<k} \gamma_{i j k} \frac{\partial f}{\partial X_{k}}+\frac{\alpha_{i j}}{f^{c-1}} .
$$

Notice that

$$
\frac{\gamma_{k i j} \partial f / \partial X_{k}}{f^{c}}=\frac{\partial}{\partial X_{k}}\left(\frac{\gamma_{k i j} /(1-c)}{f^{c-1}}\right)-\frac{*}{f^{c-1}} .
$$

Put

$$
\widetilde{\gamma_{*}}=\frac{1}{c(c-1)} \gamma_{*}
$$

Thus, we obtain

$$
\frac{b_{i j}}{f^{c}}=\sum_{k<i<j} \frac{\partial}{\partial X_{k}}\left(\widetilde{\frac{\gamma_{k i j}}{f^{c-1}}}\right)-\sum_{i<k<j} \frac{\partial}{\partial X_{k}}\left(\widetilde{\frac{\gamma_{i k j}}{f^{c-1}}}\right)+\sum_{i<j<k} \frac{\partial}{\partial X_{k}}\left(\frac{\widetilde{\gamma_{i j k}}}{f^{c-1}}\right)+\frac{\widetilde{b_{i j}}}{f^{c-1}} .
$$

Set

$$
\delta=\left(\frac{\widetilde{\gamma_{i j l}}}{f^{c-1}} \mid 1 \leq i<j<l \leq n\right) \quad \text { and } \quad \widetilde{\xi}=\left(\frac{\widetilde{b_{i j}}}{f^{c-1}} \mid 1 \leq i<j \leq n\right) .
$$

Then

$$
\xi=\phi_{3}(\delta)+\widetilde{\xi}
$$

so we have $u=\phi_{2}(\xi)=\phi_{2}(\widetilde{\xi})$. This contradicts choice of $c$. 
5.5. By Theorem 3.1 we have

$$
H_{1}\left(\partial ; R_{f}\right)=H_{1}\left(\partial ; R_{f}\right)_{-\omega}=\frac{Z_{1}\left(\partial ; R_{f}\right)_{-\omega}}{B_{1}\left(\partial ; R_{f}\right)_{-\omega}}
$$

Let $x \in H_{1}\left(\partial ; R_{f}\right)$ be nonzero. Define

$$
L(x)=\min \left\{L(\xi) \mid x=[\xi], \text { where } \xi \in Z_{1}\left(\partial, R_{f}\right)_{-\omega}\right\}
$$

Let $\xi=\left(\xi_{1}, \ldots, \xi_{n}\right)^{\prime} \in Z_{1}\left(\partial, R_{f}\right)_{-\omega}$ be such that $x=[\xi]$, so $\xi \in\left(\mathbb{K}_{1}\right)_{-\omega}$. Thus, $\xi_{i} \in R_{f}\left(+\omega_{i}\right)_{-\omega}$, so if $\xi \neq 0$, then $\xi \in R_{f}^{n} \backslash R^{n}$. It follows that $L(\xi) \geq 1$. Thus, $L(x) \geq 1$.

We now define a function

$$
\begin{aligned}
\widetilde{\theta}: H_{1}\left(\partial, R_{f}\right) & \rightarrow H_{1}(\partial(f) ; A), \\
x & \mapsto \begin{cases}\theta(\xi) & \text { if } x \neq 0, x=[\xi], \text { and } L(x)=L(\xi), \\
0 & \text { if } x=0 .\end{cases}
\end{aligned}
$$

Proposition 5.6 (with hypotheses as above). The element $\widetilde{\theta(x)}$ is independent of the choice of $\xi$.

Proof. Suppose that $x=\left[\xi_{1}\right]=\left[\xi_{2}\right]$ is nonzero and that $L(x)=L\left(\xi_{1}\right)=$ $L\left(\xi_{2}\right)=i$. Let $\left(a_{1} / f^{i}, \ldots, a_{n} / f^{i}\right)^{\prime}$ be the normal form of $\xi_{1}$, and let $\left(b_{1} / f^{i}\right.$, $\left.\ldots, b_{n} / f^{i}\right)^{\prime}$ be the normal form of $\xi_{2}$. It follows that $\xi_{1}=\xi_{2}+\delta$, where $\delta \in B_{1}\left(\partial ; R_{f}\right)_{-\omega}$. By Proposition $4.7(1)$, we get $j=L(\delta) \leq i$. Let $\left(c_{1} / f^{j}, \ldots\right.$, $\left.c_{n} / f^{j}\right)^{\prime}$ be the normal form of $\delta$. We consider two cases.

Case 1: $j<i$. Then note that $a_{k}=b_{k}+f^{i-j} c_{k}$ for $k=1, \ldots, n$. It follows that

$$
\theta\left(\xi_{1}\right)=\left[\left(\overline{a_{1}}, \ldots, \overline{a_{n}}\right)\right]=\left[\left(\overline{b_{1}}, \ldots, \overline{b_{n}}\right)\right]=\theta\left(\xi_{2}\right)
$$

Case 2: $j=i$. Then note that $a_{k}=b_{k}+c_{k}$ for $k=1, \ldots, n$. It follows that

$$
\theta\left(\xi_{1}\right)=\theta\left(\xi_{2}\right)+\theta(\delta)
$$

However, by Proposition $4.5, \theta(\delta)=0$, so $\theta\left(\xi_{1}\right)=\theta\left(\xi_{2}\right)$. Thus, $\widetilde{\theta(x)}$ is independent of choice of $\xi$. 
5.7. We now construct a filtration $\mathcal{F}=\left\{\mathcal{F}_{\nu}\right\}_{\nu \geq 0}$ of $H_{1}\left(\partial, R_{f}\right)$. Set

$$
\mathcal{F}_{\nu}=\left\{x \in H_{1}\left(\partial, R_{f}\right) \mid L(x) \leq \nu\right\}
$$

We prove the following proposition.

Proposition 5.8. We have the following:

(1) $\mathcal{F}_{\nu}$ is a $K$ subspace of $H_{1}\left(\partial ; R_{f}\right)$,

(2) $\mathcal{F}_{\nu} \supseteq \mathcal{F}_{\nu-1}$ for all $\nu \geq 1$,

(3) $\mathcal{F}_{\nu}=H_{1}\left(\partial ; R_{f}\right)$ for all $\nu \gg 0$,

(4) $\mathcal{F}_{0}=0$.

Proof. (1) Let $x \in \mathcal{F}_{\nu}$, and let $\alpha \in K$. Then by Proposition 4.7,

$$
L(\alpha x) \leq L(x) \leq \nu
$$

so $\alpha x \in \mathcal{F}_{\nu}$.

Let $x, x^{\prime} \in \mathcal{F}_{\nu}$ be nonzero. Let $\xi, \xi^{\prime} \in Z_{1}\left(\partial ; R_{f}\right)$ be such that $x=[\xi], x^{\prime}=$ $\left[\xi^{\prime}\right]$ and $L(x)=L(\xi), L\left(x^{\prime}\right)=L\left(\xi^{\prime}\right)$. Then $x+x^{\prime}=\left[\xi+\xi^{\prime}\right]$. It follows that

$$
L\left(x+x^{\prime}\right) \leq L\left(\xi+\xi^{\prime}\right) \leq \max \left\{L(\xi), L\left(\xi^{\prime}\right)\right\} \leq \nu .
$$

Note that the second inequality follows from Proposition 4.7. Thus, $x+x^{\prime} \in \mathcal{F}_{\nu}$.

(2) This is clear.

(3) Let $\mathcal{B}=\left\{x_{1}, \ldots, x_{m}\right\}$ be a $K$-basis of $H_{1}\left(\partial ; R_{f}\right)=H_{1}\left(\partial ; R_{f}\right)_{-\omega}$. Let

$$
c=\max \left\{L\left(x_{i}\right) \mid i=1, \ldots, m\right\} .
$$

We claim that

$$
\mathcal{F}_{\nu}=H_{1}\left(\partial ; R_{f}\right) \quad \text { for all } \nu \geq c .
$$

Fix $\nu \geq c$. Let $\xi_{i} \in Z_{1}\left(\partial ; R_{f}\right)_{-\omega}$ be such that $x_{i}=\left[\xi_{i}\right]$ and $L\left(x_{i}\right)=L\left(\xi_{i}\right)$ for $i=1, \ldots, m$.

Let $u \in H_{1}\left(\partial ; R_{f}\right)$. Say that $u=\sum_{i=1}^{m} \alpha_{i} x_{i}$ for some $\alpha_{1}, \ldots, \alpha_{m} \in K$. Then $u=\left[\sum_{i=1}^{m} \alpha_{i} \xi_{i}\right]$. It follows that

$$
L(u) \leq L\left(\sum_{i=1}^{m} \alpha_{i} x_{i}\right) \leq \max \left\{L\left(\xi_{i}\right) \mid i=1, \ldots, m\right\}=c \leq \nu .
$$

Here the second inequality follows from Proposition 4.7, so $u \in \mathcal{F}_{\nu}$. Thus, $\mathcal{F}_{\nu}=H_{1}\left(\partial ; R_{f}\right)$.

(4) If $x \in H_{1}\left(\partial ; R_{f}\right)$ is nonzero, then $L(x) \geq 1$. It follows that $\mathcal{F}_{0}=0$. 
5.9. Let $\mathcal{G}=\bigoplus_{\nu \geq 1} \mathcal{F}_{\nu} / \mathcal{F}_{\nu-1}$. For $\nu \geq 1$ we define

$$
\begin{aligned}
\eta_{\nu}: \frac{\mathcal{F}_{\nu}}{\mathcal{F}_{\nu-1}} & \rightarrow H_{1}(\partial(f) ; A)_{(\nu+1) \operatorname{deg} f-\omega}, \\
u & \mapsto \begin{cases}0 & \text { if } u=0, \\
\widetilde{\theta}(x) & \text { if } u=x+\mathcal{F}_{\nu-1} \text { is nonzero. }\end{cases}
\end{aligned}
$$

Proposition 5.10 (with hypotheses as above). The element $\eta_{\nu}(u)$ is independent of choice of $x$.

Proof. Suppose that $u=x+\mathcal{F}_{\nu-1}=x^{\prime}+\mathcal{F}_{\nu-1}$ is nonzero. Then $x=x^{\prime}+y$, where $y \in \mathcal{F}_{\nu-1}$. Because $u \neq 0$, we have $x, x^{\prime} \in \mathcal{F}_{\nu} \backslash \mathcal{F}_{\nu-1}$, so $L(x)=L\left(x^{\prime}\right)=$ $\nu$. Say that $x=[\xi], x^{\prime}=\left[\xi^{\prime}\right]$ and that $y=[\delta]$, where $\xi, \xi^{\prime}, \delta \in Z_{1}\left(\partial ; R_{f}\right)$ with $L(\xi)=L\left(\xi^{\prime}\right)=\nu$ and $L(\delta)=L(y)=k \leq \nu-1$. Thus, we have $\xi=\xi^{\prime}+\delta+\alpha$, where $\alpha \in B_{1}\left(\partial ; R_{f}\right)_{-\omega}$. Let $L(\alpha)=r$. Note that $r \leq \nu$.

Let $\left(a_{1} / f^{\nu}, \ldots, a_{n} / f^{\nu}\right)^{\prime}, \quad\left(a_{1}^{\prime} / f^{\nu}, \ldots, a_{n}^{\prime} / f^{\nu}\right)^{\prime}, \quad\left(b_{1} / f^{k}, \ldots, b_{n} / f^{k}\right)^{\prime}$, and $\left(c_{1} / f^{r}, \ldots, c_{n} / f^{r}\right) \prime$ be normal forms of $\xi, \xi^{\prime}, \delta$, and $\alpha$, respectively. Thus, we have

$$
a_{j}=a_{j}^{\prime}+f^{\nu-k} b_{j}+f^{\nu-r} c_{j} \quad \text { for } j=1, \ldots, n \text {. }
$$

Case 1: $r<\nu$. In this case we have $\overline{a_{j}}=\overline{a_{j}^{\prime}}$ in $A$ for each $j=1, \ldots, n$, so $\theta(\xi)=\theta\left(\xi^{\prime}\right)$. Thus, $\widetilde{\theta}(x)=\widetilde{\theta}\left(x^{\prime}\right)$.

Case 2: $r=\nu$. In this case notice that $\overline{a_{j}}=\overline{a_{j}^{\prime}}+\overline{c_{j}}$ in $A$ for each $j=$ $1, \ldots, n$, so $\theta(\xi)=\theta\left(\xi^{\prime}\right)+\theta(\alpha)$. However, $\theta(\alpha)=0$ as $\alpha \in B_{1}\left(\partial ; R_{f}\right)_{-\omega}$ (see Proposition 4.5). Thus, $\widetilde{\theta}(x)=\widetilde{\theta}\left(x^{\prime}\right)$.

Note that neither $\theta$ nor $\tilde{\theta}$ is linear. However, we prove the following.

Proposition 5.11 (with notation as above). For all $\nu \geq 1$, $\eta_{\nu}$ is $K$-linear.

Proof. Let $u, u^{\prime} \in \mathcal{F}_{\nu} / \mathcal{F}_{\nu-1}$. We first show that $\eta_{\nu}(\alpha u)=\alpha \eta_{\nu}(u)$ for all $\alpha \in K$. We have nothing to show if $\alpha=0$ or if $u=0$, so assume that $\alpha \neq 0$ and that $u \neq 0$. Say that $u=x+\mathcal{F}_{\nu-1}$. Then $\alpha u=\alpha x+\mathcal{F}_{\nu-1}$. Because $\widetilde{\theta}(\alpha x)=\alpha \widetilde{\theta}(x)$, we get the result.

Next we show that $\eta_{\nu}\left(u+u^{\prime}\right)=\eta_{\nu}(u)+\eta_{\nu}\left(u^{\prime}\right)$. We have nothing to show if $u$ or $u^{\prime}$ is zero. Next we consider the case when $u+u^{\prime}=0$. Then $u=-u^{\prime}$, 
so $\eta_{\nu}(u)=-\eta_{\nu}\left(u^{\prime}\right)$. Thus, in this case

$$
\eta_{\nu}\left(u+u^{\prime}\right)=0=\eta_{\nu}(u)+\eta_{\nu}\left(u^{\prime}\right) .
$$

Now consider the case when $u, u^{\prime}$ are nonzero and $u+u^{\prime}$ is nonzero. Say that $u=x+\mathcal{F}_{\nu-1}$ and that $u^{\prime}=x^{\prime}+\mathcal{F}_{\nu-1}$. Note that because $u+u^{\prime}$ is nonzero, $x+x^{\prime} \in \mathcal{F}_{\nu} \backslash \mathcal{F}_{\nu-1}$. Let $x=[\xi]$, and let $x^{\prime}=\left[\xi^{\prime}\right]$, where $\xi, \xi^{\prime} \in Z_{1}\left(\partial ; R_{f}\right)_{-\omega}$ and $L(\xi)=L\left(\xi^{\prime}\right)=\nu$. Then $x+x^{\prime}=\left[\xi+\xi^{\prime}\right]$. Note that $L\left(\xi+\xi^{\prime}\right) \leq \nu$ by Proposition 4.7. But $L\left(x+x^{\prime}\right)=\nu$, so $L\left(\xi+\xi^{\prime}\right)=\nu$. Let $\left(a_{1} / f^{\nu}, \ldots, a_{n} / f^{\nu}\right)^{\prime}$, $\left(a_{1}^{\prime} / f^{\nu}, \ldots, a_{n}^{\prime} / f^{\nu}\right)^{\prime}$ be normal forms of $\xi$ and $\xi^{\prime}$, respectively. Note that $\left(\left(a_{1}+a_{1}^{\prime}\right) / f^{\nu}, \ldots,\left(a_{n}+a_{n}^{\prime}\right) / f^{\nu}\right)^{\prime}$ is the normal form of $\xi+\xi^{\prime}$. It follows that $\theta\left(\xi+\xi^{\prime}\right)=\theta(\xi)+\theta\left(\xi^{\prime}\right)$. Thus, $\widetilde{\theta}\left(x+x^{\prime}\right)=\widetilde{\theta}(x)+\widetilde{\theta}\left(x^{\prime}\right)$. Therefore,

$$
\eta_{\nu}\left(u+u^{\prime}\right)=\eta_{\nu}(u)+\eta_{\nu}\left(u^{\prime}\right) .
$$

Finally we have the main result of this section.

Proof of Theorem 2. Let $\nu \geq 1$. By Proposition 5.11, we know that $\eta_{\nu}$ is a linear map of $K$-vector spaces. We now prove that $\eta_{\nu}$ is injective.

Suppose, if possible, that $\eta_{\nu}$ is not injective. Then there exists nonzero $u \in \mathcal{F}_{\nu} / \mathcal{F}_{\nu-1}$ with $\eta_{\nu}(u)=0$. Say that $u=x+\mathcal{F}_{\nu-1}$. Also, let $x=[\xi]$, where $\xi \in Z_{1}\left(\partial ; R_{f}\right)_{-\omega}$ and $L(\xi)=L(x)=\nu$. Let $\left(a_{1} / f^{\nu}, \ldots, a_{n} / f^{\nu}\right)^{\prime}$ be the normal form of $\xi$. Thus, we have

$$
0=\eta_{\nu}(u)=\widetilde{\theta}(x)=\theta(\xi)=\left[\left(\overline{a_{1}}, \ldots, \overline{a_{n}}\right)^{\prime}\right] .
$$

It follows that $\left(\overline{a_{1}}, \ldots, \overline{a_{n}}\right)^{\prime}=\psi_{2}(\bar{b})$, where $\bar{b}=\left(\overline{b_{i j}} \mid 1 \leq i<j \leq n\right)^{\prime}$. It follows that, for $l=1, \ldots, n$,

$$
\overline{a_{l}}=\sum_{i<l} \overline{b_{i l}} \frac{\partial f}{\partial X_{i}}-\sum_{l>j} \overline{b_{l j}} \frac{\partial f}{\partial X_{j}} .
$$

Then it follows that for $l=1, \ldots, n$ we have the following equation in $R$ :

$$
a_{l}=\sum_{i<l} b_{i l} \frac{\partial f}{\partial X_{i}}-\sum_{l>j} b_{l j} \frac{\partial f}{\partial X_{j}}+d_{l} f
$$

for some $d_{l} \in R$. Note that (5.11.1) is of homogeneous elements in $R$. Thus, we have the following:

$$
\frac{a_{l}}{f^{\nu}}=\frac{\sum_{i<l} b_{i l} \frac{\partial f}{\partial X_{i}}}{f^{\nu}}-\frac{\sum_{l>j} b_{l j} \frac{\partial f}{\partial X_{j}}}{f^{\nu}}+\frac{d_{l}}{f^{\nu-1}} .
$$


We consider two cases.

Case 1: $\nu \geq 2$. Set $\widetilde{b_{i j}}=-b_{i l} /(c-1)$. Then note that

$$
\frac{b_{i l} \frac{\partial f}{\partial X_{i}}}{f^{\nu}}=\frac{\partial}{\partial X_{i}}\left(\frac{\widetilde{b_{i l}}}{f^{\nu-1}}\right)-\frac{*}{f^{\nu-1}} .
$$

By (5.11.2) we have, for $l=1, \ldots, n$,

$$
\frac{a_{l}}{f^{\nu}}=\sum_{i<l} \frac{\partial}{\partial X_{i}}\left(\frac{\widetilde{b_{i l}}}{f^{\nu-1}}\right)-\sum_{l<j} \frac{\partial}{\partial X_{j}}\left(\frac{\widetilde{b_{l j}}}{f^{\nu-1}}\right)+\frac{c_{l}}{f^{\nu-1}} .
$$

Put $\xi^{\prime}=\left(c_{1} / f^{\nu-1}, \ldots, c_{n} / f^{\nu-1}\right)^{\prime}$, and put $\delta=\left(\widetilde{b_{i j}} / f^{\nu-1} \mid 1 \leq i<j \leq n\right)$. Then we have

$$
\xi=\phi_{2}(\delta)+\xi^{\prime}
$$

so we have $x=[\xi]=\left[\xi^{\prime}\right]$. This yields $L(x) \leq L\left(\xi^{\prime}\right) \leq \nu-1$. This is a contradiction.

Case 2: $\nu=1$. Note that $\xi \in\left(\mathbb{K}_{1}\right)_{-\omega}$. Thus, for $l=1, \ldots, n$ we have

$$
\frac{a_{l}}{f} \in\left(R_{f}\left(\omega_{l}\right)\right)_{-\omega}
$$

It follows that

$$
\operatorname{deg} a_{l}=\operatorname{deg} f-\sum_{k \neq l} \omega_{k}
$$

Also note that $\operatorname{deg} \partial f / \partial X_{i}=\operatorname{deg} f-\omega_{i}$. By comparing degrees in (5.11.1) we get $a_{l}=0$ for all $l$. Thus, $\xi=0$, so $x=0$. Therefore, $u=0$, a contradiction.

\section{§6. Example 0.1}

Let $R=K\left[X_{1}, \ldots, X_{n}\right]$, and let $f=X_{1}^{2}+\cdots+X_{n-1}^{2}+X_{n}^{m}$, with $m \geq 2$. Set $A=R /(f)$. In this section we compute $H_{1}\left(\partial ; H_{(f)}^{1}(R)\right)$.

6.1. We give $\omega_{i}=\operatorname{deg} X_{i}=m$ for $i=1, \ldots, n-1$, and we give $\omega_{n}=$ $\operatorname{deg} X_{n}=2$. Note that $f$ is a homogeneous polynomial in $R$ of degree $2 \mathrm{~m}$. Also note that $\omega=\sum_{k=1}^{n} \omega_{k}=(n-1) m+2$. 
6.2. First note that the Jacobian ideal $J$ of $f$ is primary to the unique graded maximal ideal of $R$. It follows that $A$ is an isolated singularity. Note that $J=\left(X_{1}, \ldots, X_{n-1}, X_{n}^{m-1}\right)$. Let $H_{i}(J ; A)$ be the $i$ th Koszul homology of $A$ with respect to $J$.

Proposition 6.3. The Hilbert series, $P(t)$, of $H_{1}(J ; A)$ is

$$
P(t)=\sum_{k=0}^{m-2} t^{2 m+2 k}
$$

Proof. It is easily verified that $X_{1}, \ldots, X_{n-1}$ is an $A$-regular sequence. Set

$$
B=A /\left(X_{1}, \ldots, X_{n-1}\right) A=\frac{K\left[X_{n}\right]}{\left(X_{n}^{m}\right)}=K \oplus K X_{n} \oplus X_{n}^{2} \oplus \cdots \oplus K X_{n}^{m-1} .
$$

Note that we have an exact sequence

$$
0 \rightarrow H_{1}(J ; A) \rightarrow B(-2(m-1)) \stackrel{X_{n}^{m-1}}{\longrightarrow} B .
$$

It follows that $H_{1}(J ; A)=X_{n} B(-2(m-1))$. The result follows.

6.4. By Theorem 2 there exists a filtration $\mathcal{F}=\left\{\mathcal{F}_{\nu}\right\}_{\nu \geq 0}$ consisting of $K$-subspaces of $H_{1}\left(\partial ; H_{(f)}^{1}(R)\right)$ with $\mathcal{F}_{\nu}=H^{n-1}\left(\partial ; H_{(f)}^{1}(R)\right)$ for $\nu \gg 0, \mathcal{F}_{\nu} \supseteq$ $F_{\nu-1}$, and $\mathcal{F}_{0}=0$ and injective $K$-linear maps

$$
\eta_{\nu}: \frac{\mathcal{F}_{\nu}}{\mathcal{F}_{\nu-1}} \longrightarrow H_{1}(\partial(f) ; A)_{(\nu+1) \operatorname{deg} f-\omega}
$$

Notice that

$$
(\nu+1) \operatorname{deg} f-\omega=(\nu+1) 2 m-(n-1) m-2=(2 \nu-n+3) m-2 .
$$

If $\eta_{\nu} \neq 0$, then by Proposition 6.3 it follows that

$$
(2 \nu-n+3) m-2=2 m+2 j \quad \text { for some } j=0, \ldots, m-2 .
$$

Thus, we obtain

$$
2 \nu m=(n-1) m+2(j+1)
$$

It follows that $m$ divides $2(j+1)$. Because $2(j+1) \leq 2 m-2$, it follows that $2(j+1)=m$. Thus, $m$ is even. 
6.5. Say that $m=2 r$. Then by (6.4.1) we have

$$
2 \nu r=(n-1) r+r
$$

so $\nu=n / 2$. It follows that $n$ is even. Furthermore, note that $\eta_{\nu}=0$ for $\nu \neq n / 2$ and that if $\nu=n / 2$ then by $(6.3) \operatorname{dim} \mathcal{F}_{n / 2} / \mathcal{F}_{n / 2-1} \leq 1$. It follows that in this case $\operatorname{dim} H_{1}\left(\partial ; H_{(f)}^{1}(R)\right) \leq 1$.

6.6. In conclusion we have the following:

(1) if $m$ is odd, then $H^{n-1}\left(\partial ; H_{(f)}^{1}(R)\right)=0$;

(2) if $m$ is even, then

(a) if $n$ is odd then $H^{n-1}\left(\partial ; H_{(f)}^{1}(R)\right)=0$,

(b) if $n$ is even then $\operatorname{dim}_{K} H^{n-1}\left(\partial ; H_{(f)}^{1}(R)\right) \leq 1$.

This proves Example 0.1.

\section{REFERENCES}

[1] J.-E. Björk, Rings of Differential Operators, North-Holland Math. Library 21, NorthHolland, Amsterdam, 1979. MR 0549189.

[2] W. Bruns and J. Herzog, Cohen-Macaulay Rings, Cambridge Stud. Adv. Math. 39, Cambridge University Press, Cambridge, 1993. MR 1251956.

[3] G. Lyubeznik, Finiteness properties of local cohomology modules (an application of D-modules to commutative algebra), Invent. Math. 113 (1993), 41-55. MR 1223223. DOI 10.1007/BF01244301.

[4] L. Ma and W. Zhang, Eulerian graded D-modules, Math. Res. Lett. 21 (2014), 149167. MR 3247047. DOI 10.4310/MRL.2014.v21.n1.a13.

[5] T. J. Puthenpurakal, De Rham cohomology of local cohomology modules, preprint, arXiv: 1302.0116v2 [math.AC].

[6] T. J. Puthenpurakal and R. B. T. Reddy, de Rham cohomology of $H_{(f)}^{1}(R)$, where $V(f)$ is a smooth hypersurface in $\mathbb{P}^{n}$, preprint, arXiv:1310.4654v1 [math.AC].

Department of Mathematics

Indian Institute of Technology Bombay

Powai, Mumbai 400076

India

tputhen@math.iitb.ac.in 